\title{
A Technique for Teaching Difficult Concepts in an Undergraduate Business Database Management Systems Course at a HBCU
}

\author{
Ingrid A. Buckley, PhD \\ Florida Gulf Coast University, USA, ibuckley@fgcu.edu
}

\begin{abstract}
This paper proposes an innovative teaching technique to help students, especially business students, understand fundamental concepts to effectively design databases. Students who are not computer science majors are not usually familiar with abstract or technical principles relating to databases. A teaching approach is introduced that initially focuses on the business side, by first discussing the traditional File Processing System (FPS) and its corresponding problems. The purpose and design of a Relational Database Management System (RDBMS) are presented along with an involved discussion of the benefits it provides. A novel teaching technique is then introduced which shows students how to transform data collected from a form into a RDBMS using a generalized classification method, visualization, familiar applications and enhanced-entity relationship models. This technique is tested using a simple exercise to compare student understanding before and after learning this technique. Students showed a marked improvement in their overall understanding of RDBM systems, and were able to determine suitable entities, attributes, primary keys and the differences between them.

Keywords-- Relational Database Management System, Entity, Attribute, Enhanced Entity-Relationship Model, Inductive learning.
\end{abstract}

Digital Object Identifier (DOI):

http://dx.doi.org/10.18687/LACCEI2016.1.1.036

ISBN: 978-0-9822896-9-3

ISSN: $2414-6390$

$14^{\text {th }}$ LACCEI International Multi-Conference for Engineering, Education, and Technology: "Engineering Innovations for Global Sustainability", 20-22 July 2016, San José, Costa Rica. 


\title{
A Technique for Teaching Difficult Concepts in an Undergraduate Business Database Management Systems Course at a HBCU
}

\author{
Ingrid A. Buckley, $\mathrm{PhD}$ \\ Florida Gulf Coast University, USA, ibuckley@fgcu.edu
}

\begin{abstract}
This paper proposes an innovative teaching technique to help students, especially business students, understand fundamental concepts to effectively design databases. Students who are not computer science majors are not usually familiar with abstract or technical principles relating to databases. A teaching approach is introduced that initially focuses on the business side, by first discussing the traditional File Processing System (FPS) and its corresponding problems. The purpose and design of a Relational Database Management System (RDBMS) are presented along with an involved discussion of the benefits it provides. A novel teaching technique is then introduced which shows students how to transform data collected from a form into a RDBMS using a generalized classification method, visualization, familiar applications and enhanced-entity relationship models. This technique is tested using a simple exercise to compare student understanding before and after learning this technique. Students showed a marked improvement in their overall understanding of RDBM systems, and were able to determine suitable entities, attributes, primary keys and the differences between them.
\end{abstract}

Keywords-- Relational Database Management System, Entity, Attribute, Enhanced Entity-Relationship Model, Inductive learning

\section{INTRODUCTION}

Technology has changed the way businesses conduct their daily tasks and processes. Most businesses have evolved in how they utilize data, most times they have to collect, query, manipulate and store data rapidly in order to provide services to their consumers. In the past, the traditional File Processing System (FPS) was widely used to carry out daily business operations. However with the rapid growth of technology, and the need to manipulate larger volumes of data rapidly, the FPS became less effective for business purposes. It is no surprise that employees who work in a business environment must also upgrade and enhances their data processing knowledge and skill set.

Today, the Relational Database Management System (RDBMS) is a staple resource in businesses of all types. Naturally, businesses want employees who are able to perform their daily duties more efficiently, using various RDBMS to manage data. A consequence of using relational database management systems (RDBMS) is that employees need proper training. Employees are a product of the education system and universities have to respond to the changes in various business

Digital Object Identifier (DOI): http://dx.doi.org/10.18687/LACCEI2016.1.1.036 ISBN: 978-0-9822896-9-3

ISSN: $2414-6390$

$14^{\text {th }}$ LACCEI International Multi-Conference for Engineering, Education, and Technology: "Engineering Innovations for Global Sustainability”, 20-22 July 2016, San José, Costa Rica. practices as well as the needs of employers. Some business degree programs now offer courses such as Business Database Management System, to educate business students on how to design a database system. As a result, instructors must also, find innovative ways to convey the principles, and concepts in developing database designs that are suitable for business purposes. This is not always an easy task given the background of business students and the interdisciplinary nature of such courses, which merge business and computer science principles. It is therefore important to define techniques and approaches to effectively teach students these needed skills.

Section 2, provides motivation and challenges business students experience in the business database management systems course. Section 3, presents some background about the students' learning challenges. Section 4 presents a discussion about the traditional file processing system and why it is no longer useful for today's business needs. Section 5 , provides an overview of relational database management systems and its advantages. Section 6, presents a technique to identify entities and attributes to create a relation database. Section 7, provides an evaluation of the approach. Section 8 discusses related work and the paper concludes with section 9 .

\section{MOTIVATION}

The Business Database Management Systems course (CSCI 345) is offered once each year to business and information technology (IT) students in the Department of Computer Science in the College of Business and Information Science at Tuskegee University presents some challenges for instructors. CSCI 345 is a staple course that most Supply Chain Management majors must take; it also serves as a prerequisite to another core course in their curriculum. Two primary challenges were experienced when teaching this course. Students did not understand how to capture data from a form, report, or survey and develop a database to store the data. In general, students found it difficult to determine entities and attributes and their differences. Based on these two challenges there was a need to find some teaching approaches to help the students overcome these challenges.

\section{STUDENT LEARNING CHALLENGES}

During the course of teaching the Business Database Management Systems (DBMS) course, it was observed that 
majority of the business students experienced the following challenges:

- Business students found it difficult to link abstract concepts to relatable examples i.e. shopping online

- Difficulty determining an entity and its corresponding attributes

- Trouble understanding the difference between an entity, attribute and an attribute instance

- Are not usually exposed to the concept of Object Oriented Design (OOD)

○ Classes $\Rightarrow$ entities

- Class properties $\Rightarrow$ attributes

- Associations (relationships) made between classes

- Become confused when the problem domain changes

Additionally, majority of the examples given in database textbooks do not typically relate to their life experiences. The typical life experience of student's center around their day to day operations, using their cellular phone, shopping in store or online, conducting research on the internet, or using various software tools. As a result, business students generally have a difficult time applying concepts taught in business database systems course, especially problems from unknown problem domains.

\section{FILE PROCESSING SySTEM}

A file processing system is a system used to store and manage data that involves each department or area within an organization having its own set of files. A file processing system stores data in separate computer files. The way files are stored and shared using FPS creates some serious problems.

\subsection{Problems}

Traditional file-processing systems introduce the following problems:

- Data redundancy and inconsistency

$\circ$ One file is copied and stored in different location and is updated by different users, thus causing inconsistencies.

- Difficulty to access data

- Only one person can access a file at any given time.

- Data isolation and multiple files and formats

- Different users may be using different file formats and software versions to edit a file making it difficult maintain the same formats. Additionally, files can only be shared when a user sends them to each other instead of allowing all users to access a file in one location.

- Integrity problems

- It will be difficult to determine which copy of a file/ document to use when files are stored in multiple locations, in different file formats and updated by different users.

- Atomicity of updates:

- It is not possible to ensure atomicity of updates when several copies of a file is being updated at the same time by different users. Because of this problem, atomicity of updates is not achievable in FPS.

- Concurrent access by multiple users

$\circ$ The fact that all users with a copy of a file can update it at the same time causes integrity problems.

- Security problems

○ Having multiple copy of a file, provides attackers with multiple access points to access a file since files are stored in different locations. Additionally, it does not restrict access to files, since all users should not have the same permission or rights to access a file.

\section{RElAtional DATABASE MANAGEMENT SYSTEM}

A file processing system is a system used to store and manage data that involves each department or area within an organization having its own set of files. Database Management Systems (DBMS) provide an organized way to utilize data. They also secure information against system failure or tampering and permit data sharing among multiple users. DBMS were developed to address the difficulties of file-processing systems as outlined in section 3.0.

A Relational Database Management System (RDBMS) stores a collection of interrelated data that allows programs/users to access the data. A relational database allows the definition of data structures, storage, retrieval operations and integrity constraints. The data and relations between them are organized in tables. A table is a collection of records and each record in a table contains the same fields.

\subsection{Advantages of RDBMS}

- Data is stored once

○ Multiple record changes are not needed

o More efficient storage

- Simple to add, delete and update data

o Referential integrity allows records in different tables having a link to each other are automatically updated reflect any changes made, thus enforcing consistency

- Easy Queries

○ The Structured Query Language (SQL) was developed to manipulate data stored in RDBMS.

- Increased Security

o RDMB allows constraints to be added to certain tables which store sensitive data, thus limiting access based on the user. 
○ RDBM also utilize views which show a snapshot view of a query, this can be used to further limit the data users are able to access.

A file processing system stores data in separate computer files. The way files are stored and shared using FPS creates some serious problems.

\section{ClassifiCATION TECHNIQUE}

A business database book written by Steven Conger [1] was used because it begins with business concepts before introducing database principles [5, 6, 7, 8]. This approach utilizes visualization, inductive learning, familiar applications that students already know and use, and incorporates a classification method that allows students to easily identify the entities and their corresponding attributes using enhanced E-R models. This technique was introduced in the Business Database Management Systems course which is offered in the Department of Computer Science in the College of Business and Information Science and students responded well. The technique is comprised of the following aspects: visualization, familiar applications, and a classification process.

\subsection{Visualization}

Visualization is used in this course to enhance learning; it helps students to grasp different and abstract concepts by studying visual objects. Sometimes students experience problems remembering the definitions and the application of concepts and they may not be able to recall what was said during a lecture. Visual aids are useful when teaching abstract concepts that students have no experience or prior knowledge of. Visualization improves students' ability to recall and apply abstract concepts more readily.

Deductive learning is the traditional way of teaching, where definitions and examples that support those definitions are used. However, students find it challenging to understand abstract ideas; therefore definitions alone are insufficient and may not provide practical application examples. Another teaching option is the use of inductive learning. In inductive learning, observations and familiar examples are used to determine a pattern. This pattern describes a generalized concept of what is being taught. Patterns are useful tools in learning and applying abstract concepts. In this course, enhanced E-R models are used to demonstrate patterns.

\subsection{Utilize Familiar Applications}

Students tend to respond positively when familiar examples and application are integrated into the classroom experience. Since the CSCI345-Business Database Management system course includes principles from computer science; it is most practical to show students how everyday systems and technologies utilize database as well as the type of business operations that they perform. One useful approach is to give assignments which allow them to create databases designs for a system they have used before. These systems can include social media applications such as Facebook, Instagram, a library, or a supermarket.

\subsection{Classification Process}

During the progression of teaching the CSCI345 course students often confused entities with attributes and vice-versa. The literature defines an entity as a singular noun and something you want to collect information about. An attribute is defined as the trait, characteristic or property associated with an entity. Despite the definitions given and countless examples provided in the course, students had a difficulty determining the difference between an entity and the attribute depending on the problem being solved. An alternative approach was identified to show the difference. This approach is presented using the idea of a concept and characteristic.

\subsubsection{Classification Process}

A concept is an abstraction from specific instances. The important step here is to determine one common classification that defines all the specific instances. This common classification will serve as the entity that you wish to collect data on. Fig. 1 and 2 provides some examples given to students to determine what the entity is given all the specific instances.

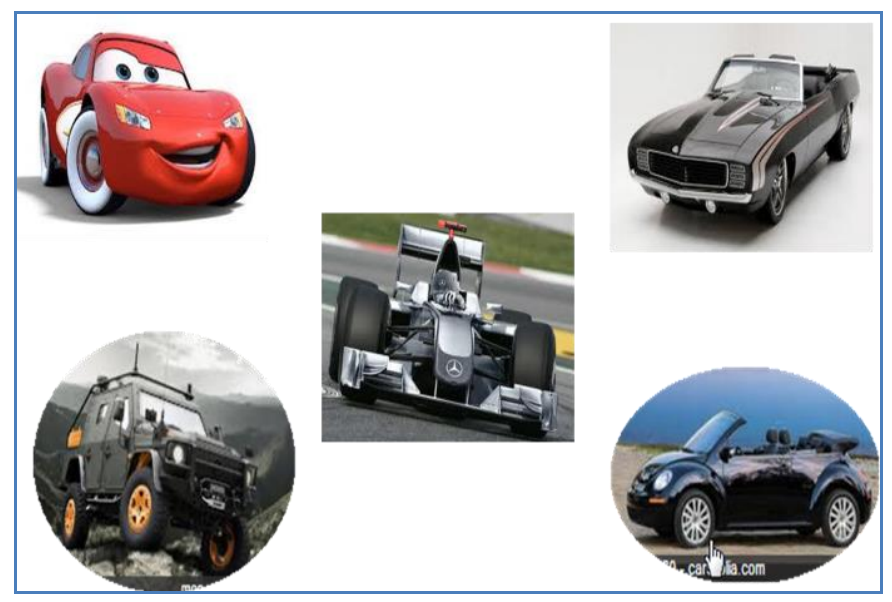

Fig. 1 Cars.

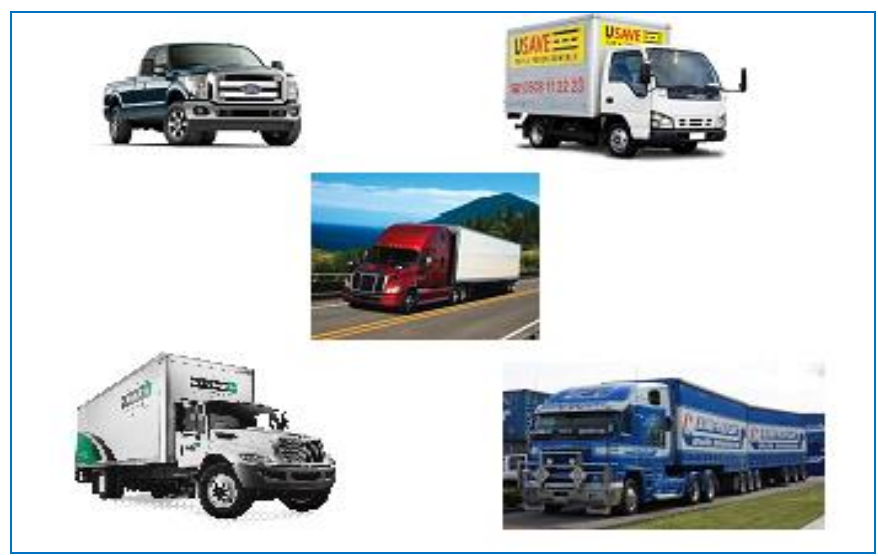

Fig. 2 Trucks

14 ${ }^{\text {th }}$ LACCEI International Multi-Conference for Engineering, Education, and Technology: "Engineering Innovations for Global Sustainability", 20-22 July 2016, San José, Costa Rica. 
Fig. 3 shows a broader group of specific instances. The students were also asked to classify the images in figure 3 to determine the entity that describes them all.

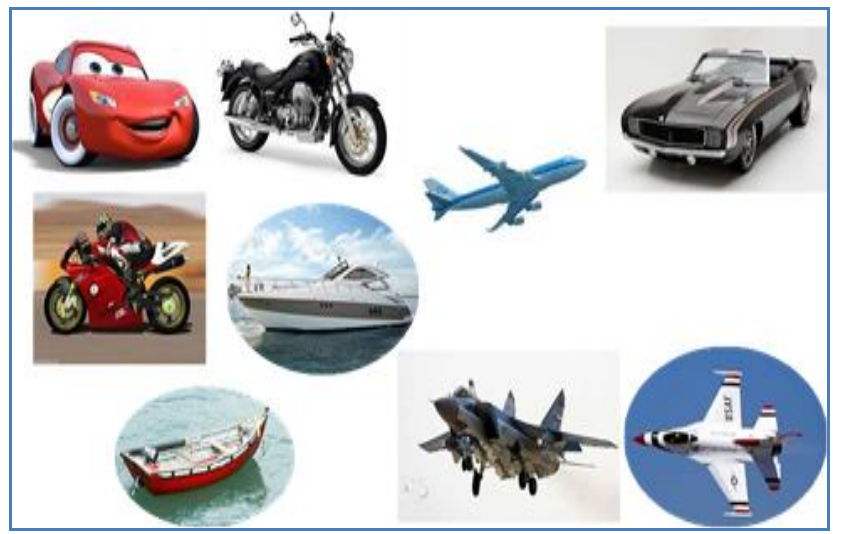

Fig. 3 Vehicles

\subsubsection{Determine Common Characteristics}

The second unit of this approach is to find the attributes: characteristics/ properties that are common amongst all the specific instances. Fig. 1 shows different types of cars, the students are asked to find all the common properties that all cars must have. These properties include: make, model, color, price and year; thus they are the attributes associated with the entity car. The same was done for the specific truck instances illustrated in Fig. 2.

Fig. 3 shows a broader group of instances which can be classified as vehicles. It is more difficult to determine the common characteristics for a broader group of instances; however the same process applies again. The process involves studying each instance and identifying all of its characteristics. The students were asked to make a list of the characteristics for each instance, they are then asked to select the characteristics that are common among all of them. Those common characteristics define the attributes of the entity. This generalized classification process, made it easier for student to identify an entity given a set of specific instances and their corresponding attributes.

\subsubsection{Enhanced Entity-Relationship Models (EER)}

The generalization technique is reinforced with the use of the enhanced entity-relationship model [3,4]. These models are used to convey patterns as explained in section 5.1. Computer Science students generally understand entities and attributes because of their CS background, however business and IT students have a difficulty understand and applying this fundamental concept. EER models [3] provide a useful tool for non CS majors to grasp the concept of entities and attributes.

EER models were developed to reflect more precisely the properties and constraints that are found in more complex databases. EER models use the concept of categorizing or generalizing between Super-types and Subtypes of entities. A car Super-type entity can have several subtypes including convertible, compact, and sedan. All of the attributes of the Super-type are also common to the Subtype. Additionally, each Subtype entity has attributes that are unique to that Subtype. Fig. 4, illustrates the vehicle supertype and the corresponding subtypes.

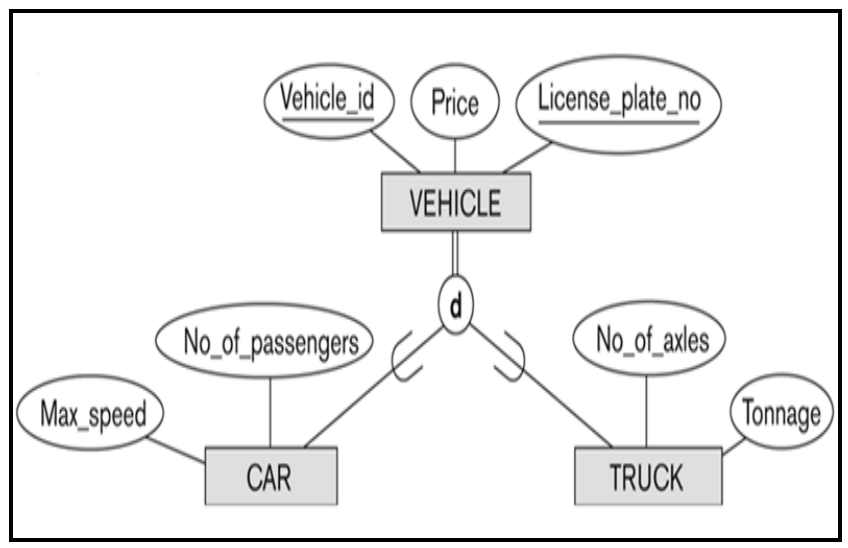

Fig. 4 Entity Relationship Model of Vehicles

In Fig. 4 [3] the vehicle supertype has two subtypes: car and truck. The attributes that are associated with the supertype vehicle are Vehicle_id, Price and License_plate_no. However the car subtype has its unique attributes: Max_speed and No_of_passengers, which only apply to cars. Similarly the truck subtype has two unique attributes: No_of_axles and Tonnage which are not shared by the supertype or the other subtype car. All subtypes inherit all the attributes of the supertype vehicle since all vehicles have those attributes in common.

\section{EvALUATION}

This technique was introduced into the CSCI345 course during the fall semester 2013. The general objective of CSCI345 course is to provide students with the background knowledge needed to analyse, evaluate, design, implement and administer database systems. Additionally, the courses teach students how to recognize the importance of databases and information resource management to support business processes. It focuses on issues and principles of managing organizational data. To test this technique a Facebook exercise was given, since most students were familiar with this social media website as described in section 6.2.

\subsection{Facebook Exercise}

Students were asked to study the Sign Up form on the Facebook website as shown in Fig. 5 (Facebook, 2004), and to identify the entities and attributes. The outcome of this exercise revealed the following:

- Most students chose one of the following as the entity: (1) Facebook, (2) the person's actual name for example, Phillip Banks and (3) Customer. 
- Similarly most students identified the following as the attributes: (1) First Name, (2) Last Name, (3) Birthday, (4) Female, (5) Male, (6) Email Contact1 and (7) E-mail Contact2.

- Most students chose one of the following as the primary key: (1) First Name, (2) Last Name, (3) Birthday.

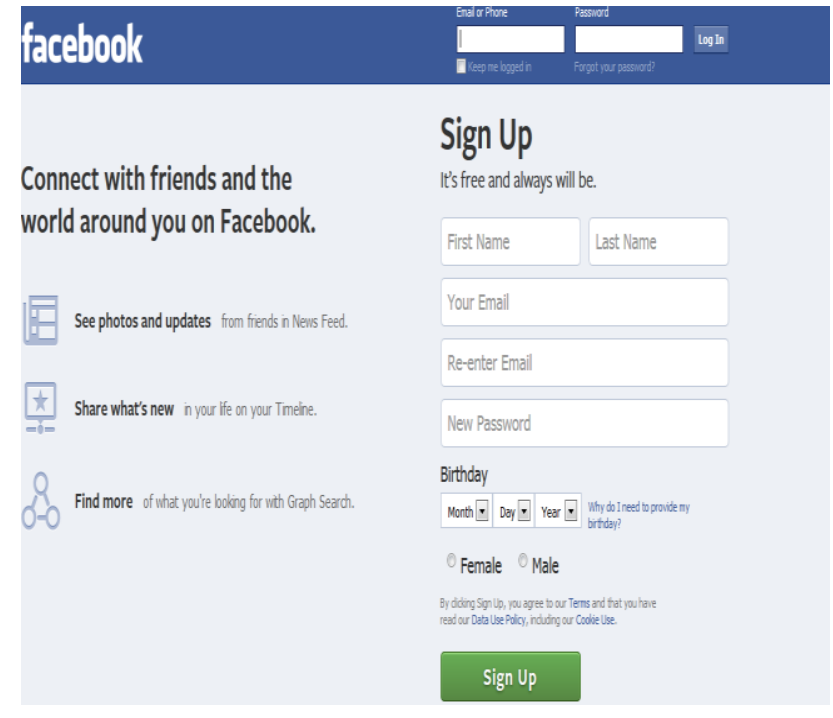

Fig. 5 Facebook Sign Up Page [2]

The answers given by students illustrates that they created attributes based on what they saw on the Facebook signup form. Generally it was observed that the students:

- Could not determine an entity type that describes the information required on the form

- Difficulty determining the difference between an entity, attribute and instance

- Created ill-matched entities and redundant attributes

- Little reasoning was done beyond the surface; and most considered female and male to be attributes because it was on the form

- Any information that has to be entered in the form twice was treated as two different attributes

- Did not consider the password

- Did not select a primary key that would uniquely identify each record

\subsection{Facebook Exercise Redone}

After the technique described in section 5 was taught the students were asked to redo (post exercise) the exercise given in section 7.1. The outcome of this exercise revealed the following:

- Most students chose one of the following as the entity: (1) User, (2) Profile.

- Similarly most students identified the following as the attributes: (1) First Name, (2) Last Name, (3) Birthday, (4) Sex, (5) E-mail and Password
- Most students chose one of the following as the primary key: (1) E-mail Address.

\begin{tabular}{|c|c|c|}
\hline \multicolumn{3}{|c|}{$\begin{array}{l}\text { FACEBOOK EXERCISE } \\
\text { OMPARISON OF STUDENT SOLUTIONS }\end{array}$} \\
\hline BEF & FORE & AFTER \\
\hline & $\begin{array}{l}\text { Entity: } \\
\text { (1) Facebook } \\
\text { (2) The peraon'a actual name } \\
\text { for example Phillip Banks and } \\
\text { (3) Customer }\end{array}$ & $\begin{array}{l}\text { Entity: } \\
\begin{array}{l}\text { (1) Uaer } \\
\text { (2) Profile }\end{array}\end{array}$ \\
\hline & $\begin{array}{l}\text { Corresponding Attributes: } \\
\text { (1) Firat Name } \\
\text { : (2) Laat Name } \\
\text { : (3) Birthday } \\
\text { : (4) Female } \\
\text { : (5) Male } \\
\text { : (6) Email Contact1 } \\
\text { (7) E-mail Contact2 }\end{array}$ & $\begin{array}{l}\text { Corresponding Attributes: } \\
\text { (1) Firat Name } \\
\text { (2) Laat Name } \\
\text { ( } \text { (3) DOB } \\
\text { - (4) Sex } \\
\text { - (5) E-mail Addreas } \\
\text { - (6) Pasaword }\end{array}$ \\
\hline & $\begin{array}{l}\text { Primary Key: } \\
\text { (1) Firat Name } \\
\text { : (2) Laat Name } \\
\text { : (3) Birthday }\end{array}$ & $\begin{array}{l}\text { Primary Key: } \\
\text { (1) E-mail Addreas }\end{array}$ \\
\hline
\end{tabular}

Fig. 6 Comparison of Facebook Exercise

This simple exercise incorporates two chief units of the technique, namely application familiarity and classifying specific instances to determine the entity and the corresponding attributes. The results in Fig. 6 show that in general students were able to improve their solution when compared to their first attempt at the Facebook exercise.

This exercise utilizes an online form, which is commonly used in most business environments. Oftentimes students will have to gather information from forms, and reports to create a database to store and manipulate data. The exercise though simple, provides a practical example of how to teach the fundamental database concepts starting with a form.

\section{RELATED WORK}

The authors in [9] present an approach for teaching database management systems using Java for an upper division database course. For this course, students must have competency in JAVA programming. In this approach, the authors create a project that is comprised of labs that are integrated in a capstone project within the students' degree program. Similarly, the authors in [10] present a strategy for teaching two database management systems courses (DBMS). The first DBMS course presents general concepts and foundation and the second course is dedicated to database management development. In their paper, they provide a summary description that outlines the topics to be covered in each course and how topics are selected and taught as the course progresses throughout the semester. They also discuss how they recruit students based on their performance in the courses to continue independent research projects. The authors describe the challenges they experienced during the teaching of these courses. The authors in [11] present a reflective write-up on teaching a database management system course to undergraduate students. Their approach utilizes a

14 ${ }^{\text {th }}$ LACCEI International Multi-Conference for Engineering, Education, and Technology: "Engineering Innovations for Global Sustainability", 20-22 July 2016, San José, Costa Rica. 
sixteen week module that outlines the topics and hands-on labs to be covered. They also obtain feedback from the students to evaluate the effectiveness of their teaching strategy. Other literature about teaching as it relates to database management systems are presented as generalized lesson plans that illustrate an outline of topics to be covered and the objectives to be achieved in a database management systems course.

In general, limited teaching approaches are available that provide detailed strategies for instructors in the teaching or relaying of database management concepts using simple and relatable exercises and examples that are adaptable to business, IT or CS undergraduate students that are not taught in a group project based format or abstract manner.

\section{CONCLUSION}

The aim of this paper is to introduce a technique that can be used for teaching undergraduate database management systems courses. This technique focuses on aiding business students by helping them to identify entities and their corresponding attributes through classifying specific instances of objects using visualization, familiarity, inductive learning, and generalization and enhanced entity relationship models. Determining an entity and its corresponding attribute is a fundamental step in designing a database. Business students initially struggled with grasping and applying this fundamental principle but showed significant improvement after learning this technique. This technique can be utilized in any database management systems courses. Instructors have a huge role to play in helping students learn, and innovative learning techniques help instructors tremendously to convey principles in a clear, practical and relatable way.

\section{ACKNOWLEDGMENT}

I would like to thank Dr. Chia Lin Chen for reviewing this paper and providing suggestions.

\section{REFERENCES}

[1] S. Conger, Hands on Database, Prentice Hall, 2010.

[2] Facebook, Facebook-Sign Up, Retrieved from: https://www.facebook.com/, 1/22/2016.

[3] P. Hensgen, Umbrello UML Modeller Handbook, unpublished.

[4] D. Bock, Enhanced e-r model and business rules, Retrieved from: http://www.siue.edu/ dbock/cmis450/4-eermodel.htm, 1/22/2016,

[5] T. Connoly, C. Begg, $4^{\text {th }}$ ed., Database Systems, Addison-Wesley, 2002.

[6] E. Elmasri, S. Navathe, $4^{\text {th }}$ ed., Fundamental of database systems, Pearson 2011.

[7] C. J. Date, $8^{\text {th }}$ ed., An Introduction to Database Systems, Addison-Wesley, 2003.

[8] R. Ramakrishnan, J. Gehrke, $3^{\text {rd }}$ ed., Database Management Systems, McGraw-Hill, 2003.

[9] M. Merzbacher, "Teaching database management systems with Java", SIGCSE, 2000 [thirty-first SIGCSE technical symposium on Computer science education (SIGCSE '00), Susan Haller (Ed.). ACM, New York, NY, USA, pp. 31-35, doi=10.1145/330908.331806].

[10] L. Yang, M. Sanver, "Teaching Database In Two Courses: Reconciling Theoretical Framework With Practical Considerations”, ASEE, 2004 [ASEE Annual Conference, Salt Lake City, Utah, 2004, June].

[11] S. Saeed, R. Aamir, Z. Mahmood, "Reflections on teaching database management systems to undergraduate students", International Journal of Education Economics and Development (IJEED), vol. II, no. 4, 2011.

14 ${ }^{\text {th }}$ LACCEI International Multi-Conference for Engineering, Education, and Technology: "Engineering Innovations for Global Sustainability", 20-22 July 2016, San José, Costa Rica. 